Int. J. Odontostomat., 9(1):101-106, 2015.

\title{
Tratamiento Odontológico Ambulatorio de Pre-Escolar con Parálisis Cerebral
}

\author{
Dental Treatment in Pre-School Outpatient with Cerebral Palsy
}

Patricia Pineda Toledo ${ }^{* * *}$; Jaime Díaz Meléndez*; Carlos Zaror Sánchez ${ }^{* *}$ \& Alejandra Jans M.*

PINEDA, T. P.; DÍAZ, M. J.; ZAROR, S. C. \& JANS, M. A. Tratamiento odontológico ambulatorio de pre-escolar con parálisis cerebral. Int. J. Odontostomat., 9(1):101-106, 2015.

RESUMEN: Los avances en medicina y en particular en pediatría han permitido sobrevivir a más niños que nacen prematuros. Aunque la mayoría de los sobrevivientes no tienen inconvenientes en su desarrollo, un significativo número de niños demuestran discapacidad en su neurodesarrollo. En los prematuros, las complicaciones y las secuelas neurológicas, aumentan con la disminución de la edad gestacional y el peso al nacer. La parálisis cerebral es la discapacidad neuromuscular más frecuente que afecta a los niños, y constituyen un grupo de alto riesgo de patologías orales. El reporte del presente caso clínico, muestra el tratamiento odontológico de un pre-escolar con parálisis cerebral, realizado en forma ambulatoria, sin premedicación ni anestesia general, sustentado en un conocimiento profundo de la patología basal médica y características individuales. La compresión de las enfermedades orales, la aplicación de técnicas de manejo conductual y la sensibilización del clínico, permitieron controlar y mejorar el deplorable estado de salud oral del pre-escolar, contribuyendo por lo tanto a una mejor calidad de vida. La promoción, la prevención y recuperación de la salud oral en los pacientes con necesidades de cuidados especiales de salud debe ser una parte integral de la odontología, para lo cual el odontopediatra debe estar integrado al equipo multidisciplinario, que busca brindar atenciones adecuadas a las necesidades de este grupo en particular.

PALABRAS CLAVE: parálisis cerebral, niños con discapacidad, salud oral.

\section{INTRODUCCIÓN}

En medicina y particularmente en pediatría, la detección e intervención de los embarazos de alto riesgo y las terapias de las Unidades de Cuidados Intensivos Neonatales, han sido pilares fundamentales en la disminución de la mortalidad perinatal e infantil, permitiendo la supervivencia en su mayoría de muchos niños que nacen prematuros, supeditado al riesgo de secuelas derivadas de la prematurez. En Chile, se ha observado una reducción sostenida de la mortalidad infantil, disminuyendo en los últimos 30 años a la décima parte y alcanzando en 2007 una tasa de 8,3 por mil nacidos vivos (Barría \& Vanegas, 2012). A nivel mundial la incidencia de parto prematuro es cercana al $10 \%$ del total de nacimientos del mundo, existiendo diferencias geográficas importantes. En nuestro país, la tasa de parto prematuro se encuentra entre el 5$6 \%$ (MINSAL, 2010).
La OMS define al recién nacido de pretérmino (RNPT), al niño que nace antes de las 37 semanas de gestación o tiene un peso inferior a $2.500 \mathrm{~g}$ al nacer (Brogard-Roth et al., 2008). Los RNPT constituyen un grupo con riesgo de padecer más enfermedades (Saavedra-Marbán et al., 2004), presentan un riesgo 17 veces mayor de morbilidad si se comparan con los recién nacidos de término. Las complicaciones de los prematuros aumentan mientras menor sea su edad gestacional o peso de nacimiento, presentando desde complicaciones leves (como hiperbilirrubinemia) hasta enfermedades graves como Enfermedad Membrana Hialina (EMH), Hemorragia Intracranea $(\mathrm{HIC})$, sepsis, Esterocolitis Necrotizante (ECN), y por cierto, secuelas neurológicas. El $50 \%$ de las anormalidades neurológicas de la infancia son atribuibles a

* Alumna Programa de Especialización en Odontopediatría Departamento de Odontopediatría y Ortodoncia, Facultad de Odontología, Universidad de La Frontera, Temuco, Chile.

Departamento de Odontopediatría y Ortodoncia, Facultad de Odontología, Universidad de La Frontera, Temuco, Chile. 
prematurez, secuelas que incluyen desde anormalidades leves de las funciones cognitivas hasta parálisis cerebral (MINSAL). Por tanto, ciertos investigadores concluyen que el número de niños con trastornos discapacitantes crónicos casi se ha triplicado en los últimos treinta años en todos los países desarrollados y sus tasas de supervivencia han aumentado de tal manera que el $90 \%$ de ellos alcanza la edad adulta (Saavedra-Marbán et al.).

La parálisis cerebral (PC) es el trastorno neuromuscular más frecuente que afecta a los niños, constituyendo en la actualidad la principal causa de discapacidad física en la infancia y se reporta una prevalencia mundial de entre 1,3 y 3,6 casos por 1.000 nacidos vivos. La PC describe un grupo de trastornos permanentes atribuido a perturbaciones no progresivas en el cerebro fetal o infantil, que afecta el desarrollo del movimiento y la postura. Los trastornos motores de la parálisis cerebral son a menudo acompañados por alteraciones en la sensación, percepción, cognición, comunicación y trastornos neurológicos (como la epilepsia), y por problemas músculo-esqueléticos secundarios. Así, numerosos problemas de salud sistémicos son comunes en los niños con PC (Du et al., 2014). La PC es una enfermedad multifactorial, los factores etiológicos más comunes citados en la literatura son hipoxia pre o post natal, recién nacido prematuro, bajo peso al nacer, infección intrauterina y sustancias tóxicas. El diagnóstico de la PC es esencialmente clínico y de acuerdo al tipo de compromiso motor, se clasifican en parálisis cerebral espástica, discinética o atáxica. Adicionalmente, de acuerdo a la ubicación de la deficiencia motora se clasifican en tetraplejía, hemiplejía, o diplejía (Katz, 2012).

La Academia Americana de Odontología Pediátrica (AAPD, 2012), define al paciente con necesidades de cuidados especiales de salud (NCES) como aquel individuo con compromiso o situación de limitación motora, sensorial, cognitiva, conductual y/o emocional que requiere de intervención, manejo médico y uso de asistencias o programas especializados en salud. La condición puede ser congénita, del desarrollo, o adquirida a través de la enfermedad, traumatismo o causa ambiental y puede imponer limitaciones en la realización de actividades diarias o sustanciales en una importante actividad de la vida. Estos individuos requieren de mayor atención, adaptación, intervención especializada y cambios en ciertas medidas que van más allá de lo que se considera un tratamiento habitual. Los individuos con NCES pueden estar en mayor riesgo de enfermedades orales, y estas enfermedades ponen en peligro aún más la salud del paciente (AAPD; Martínez et al., 2011). Este mayor riesgo es atribuido a que las infecciones orales y enfermedad periodontal son más frecuentes, y además presentan: alteraciones del esmalte, moderada a grave maloclusión y defectos congénitos craneofaciales (AL-Shehri, 2012). Ciertos medicamentos, dietas especiales, y la dificultad en el mantenimiento de la higiene diaria por parte de los padres aumentan el riesgo de caries (Kenney et al., 2008; Purohit et al., 2010; Marulanda et al., 2011). Factores sociales o económicos o incluso la falta de acceso a la atención odontológica también repercuten en la salud oral (Purohit et al.).

La atención odontológica del paciente con discapacidad representa generalmente un desafío diferente para el odontopediatra, no por el tratamiento en sí, sino por el manejo conductual de este tipo de paciente. Los pacientes NCES se presentan por lo general ansiosos, con dificultad de cooperar, y capacidad de atención limitada. Se fatigan rápidamente, de forma que los tiempos de tratamiento pueden ser limitados (Gryst \& Mount, 1999). Por consiguiente, para proporcionar un seguro y efectivo tratamiento en estos individuos, en muchos casos, es necesario el uso de anestesia general (AG). Sin embargo, en los últimos años, la necesidad de AG ha disminuido, debido al progreso en los medicamentos ansiolíticos y técnicas de sedación consciente (Stanková et al., 2011). Se reporta, que muchos de estos individuos pueden ser tratados en el ámbito odontológico de rutina, con mínimas adaptaciones especiales o con intervenciones que están dentro del alcance de la mayoría de los profesionales de la odontología (Dougherty, 2009). Además, la comprensión contemporánea de la caries, respaldada por una amplia evidencia científica, que la reconocen como un proceso dinámico de remineralización o desmineralización, brinda la posibilidad de realizar una prevención y un manejo de la enfermedad centrados en el paciente (Kidd, 2011; Pretty, 2006).

Pocos estudios en la literatura odontológica han informado sobre la rehabilitación integrada de los pacientes con PC (Katz). El reporte de este caso clínico tiene como objetivo presentar el manejo clínico odontológico ambulatorio de un pre-escolar con NCES, afectado por PC y patologías orales de gran severidad. 


\section{CASO CLINICO}

Paciente de sexo femenino 3 años y 5 meses de edad, FONASAA, residente en localidad rural aledaña a Temuco, región de La Araucanía, Chile, con patología basal médica es derivado (por primera vez), por la severidad de sus patologías orales, desde el Instituto de Rehabilitación Teletón de Temuco a la Clínica de Especialidad de Odontopediatría, de la Facultad de Odontología de la Universidad de La Frontera.

La madre firmó un documento de consentimiento informado que autoriza al odontopediatra realizar el tratamiento odontológico correspondiente a las necesidades de su hija.

De acuerdo a la anamnesis del binomio madre-hijo, los antecedentes de la historia médica indican: i) madre primigesta con preeclampsia severa y cesárea de urgencia a las 30 semanas de gestación ii) recién nacido pre-termino (30 semanas de gestación), pequeño para la edad gestacional (PEG inferior al percentil 10), muy bajo peso de nacimiento (MBPN=830 g), iii) ventilación mecánica por un día y hospitalización en neonatología durante cuatro meses, iv) leucomalacia periventricular y linfangioma peritoneal operado al mes de vida. Actualmente el diagnóstico es i) status epiléptico generalizado, convulsiones y parálisis cerebral espástica ii) retraso del desarrollo psicomotor. En tratamiento con budesonida y ácido valproico por enfermedades respiratorias recurrentes y convulsiones secundarias, respectivamente. Controlado por equipo médico interdisciplinario (Broncopulmonar, Otorrinolaringólogo, Neurológo, Oftalmólogo, Pediatra), y en tratamiento de rehabilitación en el Instituto de Rehabilitación Teletón de Temuco.

Los antecedentes odontológicos indican ausencia de lactancia materna y biberón actual (desde los tres meses de edad), sin hábitos de succión nutritiva y aseo bucal dos veces al día (sin uso pasta dental, colutorios y seda dental).

El examen clínico extraoral e intraoral, establece como diagnóstico odontológico: i) dermatitis perioral asociada a babeo persistente ii) incompetencia labial), iii) deglución infantil, iv) empuje lingual v) respiración oral, vi) dentición primaria completa con resalte aumentado, vii) gingivitis margino papilar generalizada viii) caries temprana de la infancia se- vera (ceod=15). El diagnóstico clínico (Fig. 1), fue complementado con radiografías restroalveolares, haciendo la salvedad que fueron tomadas en una etapa posterior al examen clínico inicial.

Tratamiento Integral. El periodo de tratamiento clínico se extendió desde diciembre 2011 a septiembre 2012, y fue realizado en dos etapas. De acuerdo a la condición médica del paciente y severidad de la patología oral, se consultó al médico tratante sobre los medicamentos y condición actual de salud, para garantizar la entrega segura de los servicios odontológicos. El objetivo de la primera etapa de tratamiento fue bajar los niveles de infección, controlar la progresión de la enfermedad para evitar el avance de las lesiones de caries (cavitadas y no cavitadas activas) y adaptar al pre-escolar al tratamiento odontológico. De esta manera, se instruyó a la madre en el estado oral particular, principios biológicos y agravantes de la enfermedad, con énfasis en los factores protectores: i) control de higiene por medio de cepillo e hilo dental inmediatamente posterior a las meriendas principales ii) manejo de dieta (frecuencia y oportunidad), priorizando alimentos de baja cariogenicidad (alimentos no azucarados y no adhesivos) iii) gel de clorhexidina al $0,12 \%$ durante 7 días (dos veces al día) al inicio y durante el tratamiento (uso no continuado).

La adaptación del paciente infantil fue obtenida a través de la programación de citas al inicio de la jornada de la tarde, espaciadas cada dos semanas, breves en un comienzo y luego un poco más extensas, incorporando diferentes técnicas de manejo conductual. El manejo de lesiones cavitadas activas fue a través de la técnica de restauración atraumática

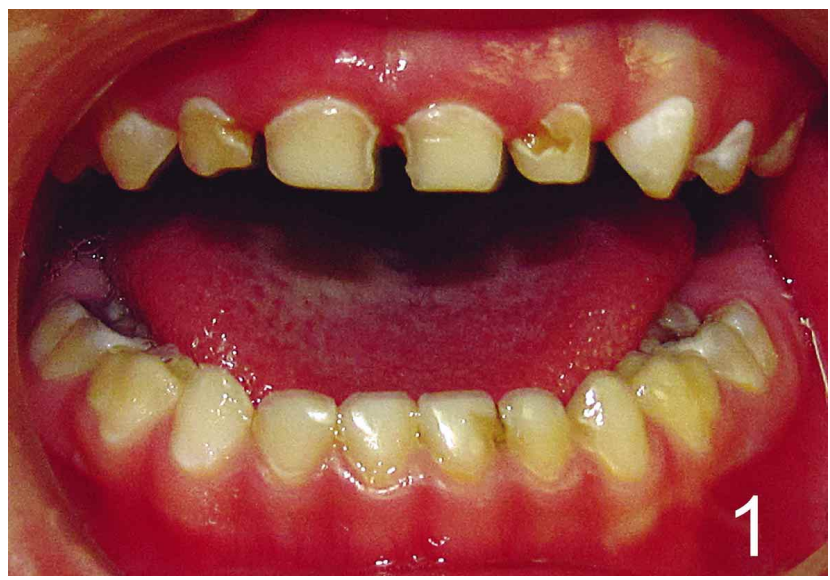

Fig. 1. Imagen del paciente antes de iniciar tratamiento. Nótese lesiones cavitadas activas y gingivitis generalizada asociada a biofilm. 
(cemento de vidrio ionómero de restauración / Ketac $^{\mathrm{TM}}$ Molar Easymix ${ }^{\circledR} 3 \mathrm{M}$ ESPE, NeussGermany) y sellante terapéutico diente 6.5 (sellante fotopolimerización Concise ${ }^{\mathrm{TM}}$ 3M ESPE, St. Paul, MN, Germany), sentando al paciente sobre el sillón dental con respaldo en posición más vertical, columna recta y piernas más elevadas, sostenidas sobre la madre ubicada a los pies del sillón. La madre hizo la contención de las manos y el apoyo de la cabeza fue dado por el propio clínico. La aplicación de barniz de flúor (Duraphat ${ }^{\circledR}$, Colgate Palmolive, Co. USA), concluyó esta primera etapa.

Después de lograr la detención de la enfermedad y una mayor adaptación del pre-escolar al tratamiento odontológico, en la segunda etapa se restauraron los molares primarios inferiores con lesiones cavitadas profundas y pulpitis reversible. Bajo anestesia local, aislamiento absoluto y técnica a cuatro manos (dos clínicos trabajando en forma simultánea), los molares 75 y 85 fueron tratados con pulpotomía con sulfato férrico al $20 \%$ (ViscoStat ${ }^{\mathrm{TM}}$ Ultradent, Products, INC) y recubrimiento pulpar directo, respectivamente. Los molares primarios mandibulares fueron obturados con cemento de vidrio ionómero de restauración (Ketac ${ }^{\mathrm{TM}}$ Molar Easymix®3M ESPE, Neuss-Germany). Finalmente, terminada esta etapa, al pre-escolar se le indicó una reevaluación cada tres meses (Fig. 2).

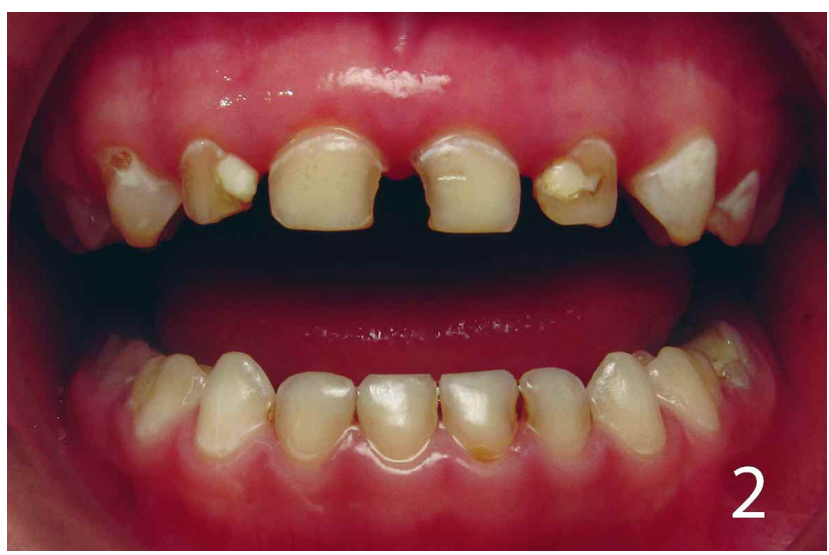

Fig. 2. Imagen al final del tratamiento. Nótese control de biofilm, lesiones de caries inactivas y salud gingival.

\section{DISCUSIÓN}

Este reporte, revela un pre-escolar, que nace prematuro y que además de presentar $\mathrm{PC}$ como patología basal médica, tiene status epiléptico generalizado, convulsiones, retraso del desarrollo psicomotor y enfermedades respiratorias recurrentes. Estos antecedentes evidencian por una parte, como los importantes avances médicos y cuidados en neonatología han disminuido la mortalidad de prematuros extremadamente pequeños (menores de 1000-1500 g), y también, como han contribuido a un aumento de la morbilidad, en los prematuros nacidos con bajo peso que sobreviven con secuelas (Saavedra-Marbán et al.).

En los niños con PC, los hallazgos orales más frecuente son una alta incidencia de caries y enfermedad periodontal, debido a la dieta (predominantemente cariogénica y pastosa), y dificultades con el control muscular para succionar, masticar y tragar (disfagia). Otros hallazgos como consecuencia de los defectos motores asociados a la PC, son la respiración bucal, trauma de tejidos (blandos y duros), bruxismo y problemas con la articulación temporomandibular (Katz; Du et al.; Nima \& Romero, 2005). En nuestro caso, la caries temprana de la infancia severa y gingivitis margino-papilar generalizada eran evidentes, como también la respiración oral e incompetencia labial. Los niños con parálisis cerebral son a menudo muy dependientes de sus cuidadores, por lo que es importante determinar el conocimiento y actitudes hacia la salud oral que tienen los padres y/o tutores (Du et al.). Por esto, la primera etapa de tratamiento consideró la educación a la madre de la condición oral de su hija (factores de riesgo de las patologías orales más prevalentes), con énfasis en el control de higiene y asesoramiento dietético, aplicando los conceptos actuales de promoción de salud, donde a la madre se le hizo comprender su rol preponderante en el cuidado, haciéndole ver su valor estratégico en el equipo de atención (Katz; Martínez et al.).

Con el propósito de reducir el riesgo de agravar la condición médica a consecuencia del tratamiento odontológico, y tomando como referencia las guía clínicas de la AAPD sobre el manejo odontológico de pacientes con NCES, se realizó una historia médica precisa y completa, además se consultó al médico tratante con respecto a los medicamentos y restricciones especiales o preparaciones, que pueden ser necesarias para garantizar la entrega segura de las prestaciones odontológicas (AAPD; Martínez et al.). El odontopediatra debe estar habituado con las características comunes de este tipo de individuos y tener in- 
formación específica de cada paciente en particular, debe tener conocimientos en psicología y experiencia práctica, debido a que en los pacientes con NCES, la angustia suele ser mayor. Debido a su inhabilidad para comunicarse o a un impedimento físico, las reacciones del discapacitado frente la situación odontológica suelen ser desiguales a las de un paciente habitual. Si el odontopediatra no está preparado para éstas conductas, pueden aparecer sentimientos de nerviosismo y molestia, pudiendo incrementar el estado de ansiedad del paciente y generar temores (Martínez et al.).

La recuperación de la salud oral de las personas con discapacidad no sólo requiere de que reciban una atención clínica de alta calidad, sino, es preponderante que tengan acceso a la atención odontológica (AL-Shehri), la cual, por supuesto requiere modificaciones de la prestación habitual. La AAPD propone que las técnicas de manejo conductual deben ser consideradas antes de la decisión de utilizar anestesia general.

El manejo de la conducta del paciente fue logrado con presencia y apoyo de la madre, mediante las técnicas de manejo conductual: i) decir-mostrarhacer ii) distracción iii) refuerzo positivo iv) control de voz v) comunicación no verbal y vi) desensibilización sistemática. Es importante resaltar que también contribuyó a su adaptación, el hecho de que el paciente asistía regularmente al Instituto de Rehabilitación Teletón, por lo cual estaba familiarizada a las prestaciones del staff médico impartidas por dicha institución, como también, contar con una madre receptiva e informada de los antecedentes médicos de su hija, acostumbrada a recibir instrucciones otorgadas por los diferentes profesio- nales. Para las lesiones cavitadas y activas se aplicó el tratamiento de restauración atraumático (TRA) y así las restauraciones se realizaron sin premedicación ni anestesia local. Esta opción de tratamiento se basó en los conceptos actuales de odontología minimamente invasiva, que establece que la eliminación parcial de tejido de dentina cariada combinado con el sellado de la cavidad, detiene el proceso de la lesión. EI TRA consideró aislamiento relativo (uso de rollos de algodón), eliminación parcial de la dentina cariada, y restauración con cemento de vidrio de ionómero (Ketac ${ }^{\mathrm{TM}}$ Molar Easymix ${ }^{\circledR} 3 \mathrm{M}$ ESPE, Neuss-Germany). Las principales ventajas del cemento de vidrio ionómero de vidrio en estas circunstancias, incluyen la adhesión a esmalte y dentina y una liberación de flúor continuo que parece ser suficiente para reducir o inhibir el biofilm (Gryst \& Mount).

Finalmente, podemos concluir que dentro de los pacientes con NECS, la PC es la discapacidad neuromuscular más frecuente que afecta a los niños. Que el manejo de estos niños depende fundamentalmente: i) de la capacidad del odontopediatra para establecer una relación y formar vínculos con el paciente, la familia y el cuidador, ii) entender y conocer en profundidad la condición del niño que se está tratando y iii) del uso apropiado de técnicas de manejo de conducta, basados en el nivel de comprensión del paciente. Sin embargo, es importante destacar que frente al eminente riesgo de enfermedades orales en los pacientes con NCES, se requiere imperiosamente que el odontopediatra, esté integrado al equipo multidisciplinario de rehabilitación, de tal manera que la intervención del especialista sea en etapas mucho más tempranas, cuando las patologías orales no se han establecido.

PINEDA, T. P.; DÍAZ, M. J.; ZAROR, S. C. \& JANS, M. A. Dental treatment in pre-school outpatient with cerebral palsy. Int. J. Odontostomat., 9(1):101-106, 2015.

ABSTRACT: Advances in medicine, particularly in pediatric medicine, have enabled more premature babies to survive. While the majority of the survivors present trouble-free development, a significant number of all children present disabilities in their neural development. In premature babies, the neurological complications and consequences increase inversely with the gestational age and weight at birth. Cerebral palsy is the most common neuromuscular disability affecting these children, and as a group they also present a high risk of oral pathologies. The report of the present clinical case describes the dental treatment of a pre-school child with cerebral palsy, in outpatient conditions and without either pre-medication or general anaesthesia, based on a profound knowledge of the basic medical pathology and individual characteristics. The understanding of oral disease, the use of behavioral management techniques and raising awareness in the clinic, enabled the child's deplorable state of oral health to be controlled and improved, thus contributing to a better quality of life. Preventative measures and the promotion and recovery of oral health in patients requiring special health care must be an integral part of dentistry. This requires the dental-pediatrician to form part of a multidisciplinary team specializing in providing proper treatment for the needs of this particular group.

KEY WORDS: cerebral palsy, disabled children, oral health. 


\section{REFERENCIAS BIBLIOGRÁFICAS:}

AL-Shehri, S. A. M. Access to dental care for persons with disabilities in Saudi Arabia (Caregivers' perspective). J. Disabil. Oral Health, 13(2):51-61, 2012.

American Academy of Pediatric Dentistry (AAPD). Guideline on management of dental patients with special health care needs. Clinical Guidelines - Reference Manual, 36(6):161-6, 2012. Disponible en: http://www.aapd.org/ media/Policies_Guidelines/G_SHCN.pdf

Barría, P. R. M. \& Vanegas L.J. Aproximaciones metodológicas al estudio de la mortalidad infantil en Chile. Rev. Chil. Pediatr., 83(1):33-41, 2012.

Brogardh-Roth, S.; Stjernqvist, K. \& Matsson, L. Dental behavioural management problems and dental caries prevalence in 3- to 6-year-old Swedish children born preterm. Int. J. Paediatr. Dent., 18(5):341-7, 2008.

Dougherty, N. The dental patient with special needs: a review of indications for treatment under general anesthesia. Spec. Care Dentist., 29(1):17-20, 2009.

Du, R. Y.; McGrath, C. P.; Yiu, C. K. \& King, N. M. Oral health behaviors of preschool children with cerebral palsy: a case-control community-based study. Spec. Care Dentist. 34(6):298-302, 2014.

Gryst, M. E. \& Mount, G. J. The use of glass ionomer in special needs patients. Aust. Dent. J., 44(4):268-74, 1999.

Katz, C. R. Integrated approach to outpatient dental treatment of a patient with cerebral palsy: a case report. Spec. Care Dentist., 32(5):210-7, 2012.

Kenney, M. K.; Kogan, M. D. \& Crall, J. J. Parental perceptions of dental/oral health among children with and without special health care needs. Ambul. Pediatr., 8(5):312-20, 2008.

Kidd, E. The implications of the new paradigm of dental caries. J. Dent., 39 Suppl. 2:S3-8, 2011.

Martínez, M. H. R.; Treviño, A. M. G. \& Rivera, S. G. Guía para el cuidado de la salud oral en pacientes con necesidad de cuidados especiales de salud en México. Rev. A. D. M., 68(5):222-8, 2011.

Marulanda, J.; Betancur, J. D.; Espinosa, S.; Gómez, J. L. \& Tapias, A. La salud oral en discapacitados. Rev. C. E. S. Odontol., 24(1):71-6, 2011.

Ministerio de Salud (MINSAL). Guía Clínica Prevención Parto Prematuro. Santiago de Chile, Ministerio de Salud
(MINSAL), 2010. Disponible en: http://web.minsal.cl/portal/url/item/721fc45c972f9016e04001011f0113bf.pdf

Nima, B. G. \& Romero, V. M. R. Tratamiento odontopediátrico integral en parálisis cerebral. Reporte de un caso. Odontol. Sanmarquina, 8(2):25-30, 2005.

Purohit, B. M.; Acharya, S. \& Bhat, M. Oral health status and treatment needs of children attending special schools in South India: a comparative study. Spec. Care Dentist., 30(6):235-41, 2010.

Pretty, I. A. Caries detection and diagnosis: novel technologies. J. Dent., 34(10):727-39, 2006.

Saavedra-Marbán, G.; Planells del Pozo, P. \& RuizExtremera, A. Patología orofacial en niños nacidos en condiciones de alto riesgo. Estudio piloto. R. C. O. E., 9(2):151-8, 2004.

Stanková, M.; Bucek, A.; Dostálová, T.; Ginzelová, K.; Pacáková, Z. \& Seydlová, M. Patients with special needs within treatment under general anesthesia - metaanalysis. Prague Med. Rep., 112(3):216-25, 2011.

Dirección para Correspondencia:

Patricia Pineda Toledo

Manuel Montt \#112

Facultad de Odontología

Universidad de La Frontera

Temuco, Chile.-

Fono/Fax: 56-45-2325776

Email: patricia.pineda@ufrontera.cl

Recibido : 19-06-2014

Aceptado: 24-09-2014 